\title{
12. Cardiología preventiva
}

\subsection{Baremo e índice (TG/HDL) como marcadores de riesgo cardiovascular en población mexicana}

Meaney Eduardo,* Martínez-Marroquín Yolanda, ${ }^{\ddagger}$ Meaney Alejandra, ${ }^{\S}$ Samaniego-Méndez Virginia, ${ }^{\S}$ Nájera Nayelli,* Ceballos-Reyes Guillermo* * Escuela Superior de Medicina, Instituto Politécnico Nacional. ${ }^{*}$ Hospital Regional de Toluca, ISSEMyM. ${ }^{\S}$ Hospital Regional $1^{0}$ Octubre, ISSSTE. Tipo de estudio: Estudio pronóstico

Introducción: Uno de los obstáculos que dificulta el control de las enfermedades ateroscleróticas es la falta de una escala de estimación de riesgo cardiovascular (CV), acorde con las características de nuestra población. El propósito del estudio fue generar un baremo de riesgo a partir de los datos del estudio Lindavista de prevención primaria en población adulta de la Ciudad de México. Objetivo: Este baremo, que incluye características de nuestra población, se comparó con el calculador del Colegio Americano de Cardiología, con la Asociación Americana de corazón (ACC/AHA) y con el cociente TG/HDL. Metodología: En la cohorte $(\mathrm{N}=2,602)$ se estudiaron las variables: edad, tabaquismo, índice de masa corporal, circunferencia abdominal, presiones arteriales sistólica y diastólica, glucemia en ayuno y perfil de lípidos. La suma de estas variables graduadas de 0 a 3 de acuerdo con la severidad, «puntaje Lindavista», se comparó con el riesgo absoluto del baremo ACC/AHA y con el cociente TG/HDL. Resultados: La correlación lineal entre ambos baremos es muy alta $(r=0.94)$. Ambos coinciden en identificar a los pacientes de bajo y muy alto riesgo, pero el calculador ACC/ AHA subestima el riesgo moderadamente alto. El índice TG/ HDL, que señala resistencia a la insulina y dislipidemia aterogénica, tiene altísima correlación lineal con ambas escalas $(r=0.94$ y 0.97). Discusión y conclusiones: La escala de riesgo CV apropiada a mexicanos debe forzosamente incluir adiposidad y triglicéridos. El índice TG/HDL es un marcador sencillo y confiable del riesgo CV en nuestra población.

\subsection{Factores de riesgo cardiovascular en artritis psoriásica comparado con artritis reumatoide}

Azpiri López José Ramón, Galarza Delgado Dionicio Ángel, Colunga Pedraza Iris Jazmín, Frausto Lerma Paola Fernanda, Rodríguez Romero Alejandra Berenice, Guajardo Jáuregui Natalia, Zárate Salinas Itzel Corina

Hospital Universitario «Dr. José Eleuterio González».

Tipo de estudio: Estudio pronóstico

Introducción: El riesgo cardiovascular (RCV) incrementado es característico en enfermedades reumatológicas, como en artritis psoriásica (APs) y artritis reumatoide (AR), donde la inflamación es crucial. Dada la coexistencia de involucro cutáneo y articular en APs, hay mayor inflamación, y es posible que el RCV sea mayor. Pocos estudios han reportado la comparación entre factores de RCV en APs y AR. Objetivo: Comparar la prevalencia de factores de riesgo cardiovascular y comorbilidades en pacientes con APs, AR y controles. Metodología: Estudio transversal. Pacientes entre 40-75 años, que cumplieron los criterios ACR/EULAR 2010 para AR y pacientes con APs que cumplieron criterios CASPAR y que asistieron a la Clínica de Cardio-Reuma durante 2018-2019, fueron seleccionados al azar y comparados con controles. El análisis descriptivo fue realizado con frecuencias (\%) y mediana (q25-q75). Comparaciones con $\chi^{2}$ y Kruskal-Wallis. Resultados: Se

Tabla 12.2.1: Características demográficas.

\begin{tabular}{|c|c|c|c|c|}
\hline & $\begin{array}{c}\text { APs }(n=95) \\
\text { n (\%) }\end{array}$ & $\begin{array}{c}\mathrm{AR}(\mathrm{n}=95) \\
\mathrm{n}(\%)\end{array}$ & $\begin{array}{c}\text { Controles }(\mathrm{n}=95) \\
\mathrm{n}(\%)\end{array}$ & $\mathbf{p}$ \\
\hline Edad (años), media \pm DE & $53.26 \pm 11.26$ & $57.48 \pm 9.59$ & $53.06 \pm 7.21$ & 0.001 \\
\hline Mujeres & $56(58.9)$ & $88(92.6)$ & $91(95.8)$ & $<0.001$ \\
\hline Duración de la enfermedad (años), mediana (q25-q175) & $4(2-8)$ & $9.91(3.75-15.91)$ & - & NS \\
\hline DAS28-PCR, media \pm DE & $2.47 \pm 1.11$ & $2.31 \pm 1.12$ & - & NS \\
\hline Índice de masa corporal $\left(\mathrm{kg} / \mathrm{m}^{2}\right)$, mediana (q25-q75) & $29.18(26.18-31.64)$ & $27.46(25.67-30.84)$ & $27.58(24.97-30.81)$ & 0.077 \\
\hline Índice cintura cadera, media $\pm \mathrm{DE}$ & $0.96 \pm 0.099$ & $0.91 \pm 0.059$ & $0.88 \pm 0.064$ & $<0.001$ \\
\hline Presión arterial aumentada $(\geq 130)$ & $43(45.3)$ & $46(48.4)$ & $26(27.4)$ & 0.004 \\
\hline Sobrepeso & $42(44.2)$ & $44(46.3)$ & $41(43.2)$ & NS \\
\hline Obesidad & $34(35.8)$ & $26(27.4)$ & $29(30.5)$ & NS \\
\hline Tabaquismo activo & $22(23.2)$ & $9(9.5)$ & $20(21.1)$ & 0.012 \\
\hline Hipertensión & $33(34.7)$ & 39 (41.1) & $22(23.2)$ & 0.029 \\
\hline Dislipidemia & 37 (38.9) & $28(29.5)$ & $27(28.4)$ & NS \\
\hline Arritmias & 0 & $3(3.2)$ & $1(1.1)$ & NS \\
\hline Diabetes mellitus & 18 (18.9) & 18 (18.9) & $15(15.8)$ & NS \\
\hline Metotrexato & $63(66.3)$ & $76(80.0)$ & - & 0.028 \\
\hline Prednisona & 17 (17.9) & 58 (61.1) & - & $<0.001$ \\
\hline Biológicos & $26(27.4)$ & $8(8.4)$ & - & 0.003 \\
\hline
\end{tabular}


incluyeron 285 sujetos. Características demográficas (Tabla 12.2.1). La hipertensión fue más prevalente en APs (34.7\%) y AR (41.1\%), comparado con controles $(23.2 \%)(p=0.029)$. Mayor tensión arterial en APs (45.3\%) que controles $(27.4 \%)(p=0.004)$. Menor prevalencia de tabaquismo activo en AR (9.5\%) comparado con APs (23.2\%) y controles $(21.1 \%)(p=0.012)$. Presencia de un mayor índice de masa corporal (IMC) entre AR y APs ( $p=0.029)$. Mayor índice cintura-cadera (ICC) en APs y AR comparado con controles ( $p<0.001)$. Discusión y conclusiones: Existe alta prevalencia de factores de RCV en APs. La evaluación sistematizada y multidisciplinaria de factores de RCV debe formar parte del manejo convencional de pacientes con APs.

\subsection{Placa carotídea en mujeres postmenopáusicas con artritis reumatoide: un estudio de casos y controles}

Azpiri López José Ramón, Rodríguez Romero Alejandra Berenice,

Lugo Pérez Salvador, Guajardo Jáuregui Natalia, Reyes Soto Mayra Alejandra, Colunga Pedraza Iris Jazmín, Galarza Delgado Dionicio Ángel

Hospital Universitario «Dr. José Eleuterio González»

Tipo de estudio: Estudio pronóstico

Introducción: La enfermedad cardiovascular (ECV) es la principal causa de muerte en el mundo y es más frecuente en mujeres postmeno- páusicas. Se ha encontrado que existe $4-5$ veces más probabilidades de placa carotídea en comparación con mujeres premenopáusicas. La artritis reumatoide (AR) se asocia con un aumento de 1.5 veces riesgo de eventos coronarios fatales, en comparación con la población general. Objetivo: Determinar la prevalencia de placa carotídea y grosor de la íntima media (GIMc) en pacientes posmenopáusicas con y sin AR. Metodología: Estudio transversal. Setenta pacientes con AR (criterios ACR/EULAR 2010) y postmenopausia y 69 controles correspondientes (sin AR). Se excluyeron pacientes con antecedentes de ECV, histerectomía y terapia de reemplazo hormonal. Se realizó ultrasonido carotídeo. Análisis descriptivo con medias, mediana y comparaciones con $\chi^{2}$. Regresión logística binaria para evaluar aumento del GIMc con factores de riesgo cardiovascular y AR. Resultados: Características demográficas (Tabla 12.3.1). La aterosclerosis carotídea derecha fue el doble $(25.7 \%$ contra $11.6 \%, p=0.033)$ y un aumento del GIMc fue más alto en pacientes con AR postmenopáusicas, comparado con controles (54.3\% contra $27.5 \%$ ) (Tabla 12.3.2). La regresión binaria mostró que tener $\mathrm{AR}$ aumenta tres veces el riesgo de tener $\mathrm{GIMc} \geq 0.9 \mathrm{~mm}$ cuando se ajusta a la edad, hipertensión, dislipidemia y tabaquismo activo RM 3.125, IC 95\% (1.54-6.39) ( $p=$ 0.004). Discusión y conclusiones: Las pacientes postmenopáusicas con AR presentaron mayor prevalencia de marcadores subclínicos de aterosclerosis comparado con pacientes postmenopáusicas sin $A R$, por lo que tienen más riesgo de desarrollar una ECV.

Tabla 12.3.1: Características demográficas.

\begin{tabular}{|c|c|c|c|}
\hline & $\begin{array}{c}\text { Artritis reumatoide }(\mathrm{n}=70) \\
\mathrm{n}(\%)\end{array}$ & $\begin{array}{c}\text { Controles }(\mathrm{n}=69) \\
\mathrm{n}(\%)\end{array}$ & $\mathbf{p}$ \\
\hline Edad, media $\pm \mathrm{DE}$ & $56.0 \pm 6.48$ & $55.04 \pm 5.38$ & 0.346 \\
\hline DM2, & $14(20.0)$ & $8(11.6)$ & 0.175 \\
\hline HTA & $26(37.1)$ & $19(27.5)$ & 0.226 \\
\hline Dislipidemia & $23(32.9)$ & $21(30.4)$ & 0.759 \\
\hline Tabaquismo activo & $5(7.1)$ & $14(20.3)$ & 0.012 \\
\hline Duración de la enfermedad mediana (q25-q75) & $10.32(4.5-15.3)$ & - & - \\
\hline IMC, mediana (q25-q75) & $28.55(26.57-31.18)$ & $28.19(25.36-31.84)$ & 0.459 \\
\hline
\end{tabular}

DM2 = diabetes mellitus 2; HTA = hipertensión arterial; IMC = índice de masa corporal.

Tabla 12.3.2: Características del ultrasonido carotídeo.

\begin{tabular}{|c|c|c|c|}
\hline & $\begin{array}{c}\text { Artritis reumatoide }(\mathrm{n}=70) \\
\mathrm{n}(\%)\end{array}$ & $\begin{array}{c}\text { Controles }(n=69) \\
n(\%)\end{array}$ & $\mathbf{p}$ \\
\hline Presencia de placa & $24(34.3)$ & $17(24.6)$ & 0.212 \\
\hline Placa bilateral & $11(15.7)$ & $4(5.8)$ & 0.060 \\
\hline Placa carotídea derecha & $18(25.7)$ & $8(11.6)$ & 0.033 \\
\hline Placa carotídea izquierda & $18(25.7)$ & $13(18.8)$ & 0.330 \\
\hline $\mathrm{GIMc} \geq 0.9 \mathrm{~mm}$ & $38(54.3)$ & $19(27.5)$ & 0.001 \\
\hline GIMc bilateral $0.9 \mathrm{~mm}$ & $18(25.7)$ & $4(5.8)$ & 0.001 \\
\hline GIMc derecha $0.9 \mathrm{~mm}$ & $25(35.7)$ & $10(14.5)$ & 0.004 \\
\hline GIMc izquierda 0.9 mm & $31(44.3)$ & $15(21.0)$ & 0.005 \\
\hline \multicolumn{4}{|l|}{ Aspecto de la placa } \\
\hline Homogénea & $19(27.1)$ & 15 (21.7) & 0.459 \\
\hline Heterogénea & $1(1.4)$ & $3(4.3)$ & 0.304 \\
\hline Mixta & $3(4.3)$ & $0(0.0)$ & 0.245 \\
\hline
\end{tabular}




\subsection{Duración diaria de sueño y riesgo cardiovascular en pacientes con artritis reumatoide}

Azpiri López José Ramón, Loya Acosta Julieta,

Gutiérrez Gándara Pablo, Zárate Salinas Itzel Corina, Lugo Pérez Salvador, Colunga Pedraza Iris Jazmín, Galarza Delgado Dionicio Ángel

Hospital Universitario «Dr. José Eleuterio González».

Tipo de estudio: Estudio pronóstico

Introducción: La reducción en la duración del sueño se ha relacionado con un mayor riesgo de enfermedad cardiovascular (ECV) y muerte. Los pacientes con artritis reumatoide (AR) tienen un mayor riesgo de ECV, al igual que en otros padecimientos dolorosos crónicos, se ha reportado una mala calidad del sueño, fatiga y sensación de falta de descanso después del sueño. Objetivo: Determinar, mediante autoevaluación, la prevalencia de la duración del sueño diario total, siestas diurnas y calidad del sueño en pacientes con AR y su asociación con factores de riesgo cardiovascular. Metodología:
Estudio observacional, transversal. Se incluyeron pacientes con AR y controles entre 40-75 años. La duración y calidad del sueño, siestas diurnas y conciencia de higiene del sueño se evaluaron con un cuestionario autoevaluado. Las asociaciones entre la duración estimada del sueño ( $<6$ horas), factores de riesgo cardiovascular y el diagnóstico de AR se evaluó mediante un análisis de regresión binaria. Resultados: Se incluyeron 217 pacientes. La duración estimada del sueño ( $<6$ horas) en AR fue 20.6 vs $8.7 \%$ ( $p=0.012)$. La presencia de ronquidos fue mayor en el grupo control $9.6 \%(p$ $=0.047$ ). Padecer AR predispone un $60 \%$ más la probabilidad de dormir $<6$ horas 0.40, IC 95\% (0.17-0.92) ( $p=0.031)$. Pacientes con duración estimada de sueño $(<6$ horas) tuvieron mayor prevalencia de hipertensión 51.6 vs 48.4\% ( $p=0.022$ ). Discusión y conclusiones: Menor tiempo de sueño en AR se asocia con hipertensión y mayor riesgo de ECV. Es imprescindible evaluar los patrones de sueño e identificar individuos de mayor riesgo para intervenciones oportunas.

Tabla 12.4.1: Factores de riesgo cardiovascular y características del sueño.

$\begin{array}{lcc} & \text { Artritis reumatoide } \mathbf{( n = 1 0 2 )} & \begin{array}{c}\text { Controles }(\mathbf{n}=\mathbf{1 1 5}) \\ \mathbf{n}(\mathbf{\%})\end{array} \\ & \mathbf{n}(\mathbf{\%}) & 57.2 \pm 10.0 \\ \mathbf{p}\end{array}$

Tabla 12.4.2: Riesgo cardiovascular en pacientes con artritis reumatoide según el tiempo estimado de sueño.

\begin{tabular}{|c|c|c|c|}
\hline & $\begin{array}{c}6 \text { horas }(n=21) \\
n(\%)\end{array}$ & $\begin{array}{c}>6 \text { horas }(n=81) \\
n(\%)\end{array}$ & $\mathbf{p}$ \\
\hline Sexo femenino & $21(100.0)$ & $72(88.9)$ & 0.110 \\
\hline $\mathrm{IMC}$, media $\pm \mathrm{DE}$ & $27.57 \pm 3.15$ & $26.75 \pm 6.5$ & 0.580 \\
\hline Peso normal & $4(25.0)$ & $25(32.5)$ & 0.099 \\
\hline Sobrepeso & $8(50.0)$ & 33 (42.9) & 0.826 \\
\hline Obesidad & $4(25.0)$ & $19(24.7)$ & 0.699 \\
\hline Tabaquismo & $3(14.3)$ & $9(11.1)$ & 0.553 \\
\hline Diabetes mellitus & $6(28.6)$ & $9(11.1)$ & 0.044 \\
\hline Hipercolesterolemia & $5(25.0)$ & $13(16.0)$ & 0.349 \\
\hline Hipertrigliceridemia & $7(33.3)$ & $7(8.6)$ & 0.003 \\
\hline Hipertensión & $11(52.4)$ & $29(35.8)$ & 0.166 \\
\hline Sedentarismo & $15(71.4)$ & $45(55.6)$ & 0.188 \\
\hline Presencia de ronquidos & $1(4.8)$ & $2(2.5)$ & 0.579 \\
\hline
\end{tabular}

IMC = índice de masa corporal. 
Tabla 12.5.1: Características clínicas y demográficas.

\begin{tabular}{|c|c|c|c|}
\hline & $\begin{array}{c}\text { APs }(n=69) \\
n(\%)\end{array}$ & $\begin{array}{c}\text { Controles }(n=69) \\
n(\%)\end{array}$ & $\mathbf{p}$ \\
\hline Edad, media \pm DE & $53.58 \pm 10.94$ & $53.86 \pm 7.31$ & NS \\
\hline Mujeres & $38(55.1)$ & $59(85.5)$ & $<0.001$ \\
\hline Obesidad & $26(37.7)$ & $28(40.6)$ & NS \\
\hline Diabetes mellitus 2 & $14(20.3)$ & $9(13.0)$ & NS \\
\hline Hipertensión arterial & 27 (39.1) & $19(27.5)$ & NS \\
\hline Dislipidemia & $29(42.0)$ & $24(34.8)$ & NS \\
\hline Tabaquismo activo & $15(21.7)$ & $12(17.4)$ & NS \\
\hline FRS-lípidos, mediana (q25-q75) & $5(2.4-13.75)$ & $6.7(3.73-10.85)$ & NS \\
\hline Reclasificación por PC & $17(24.6)$ & $15(21.7)$ & NS \\
\hline Reclasificación por GIMc & $8(11.6)$ & $1(1.4)$ & 0.016 \\
\hline FRS-IMC, mediana (q25-q75) & $11.9(4.78-21.83)$ & $9(4.6-13.3)$ & NS \\
\hline Reclasificación por PC & $15(21.7)$ & $13(18.8)$ & NS \\
\hline Reclasificación por GIMc & $3(4.3)$ & $1(1.4)$ & NS \\
\hline ACC/AHA 2013, mediana (q25-q75) & $6.6(1.7-16.55)$ & $2.9(1.53-5.03)$ & 0.001 \\
\hline Reclasificación por PC & $14(20.3)$ & $16(23.2)$ & NS \\
\hline Reclasificación por GIMc & $6(8.7)$ & $1(1.4)$ & NS \\
\hline Duración de la enfermedad, mediana (q25-q75) & $5(2.5-8.0)$ & - & - \\
\hline Metotrexato & $46(66.7)$ & - & - \\
\hline Biológicos & $23(33.3)$ & - & - \\
\hline DAS28-VSG, media \pm DE & $3.74 \pm 1.477$ & - & - \\
\hline DAS28-PCR, media \pm DE & $2.43 \pm 1.088$ & - & - \\
\hline DAPSA, mediana (q25-q75) & $35(27.5-58.5)$ & - & - \\
\hline
\end{tabular}

\subsection{Reclasificación de riesgo cardiovascular mediante ultrasonido carotídeo en artritis psoriásica}

Azpiri López José Ramón, Gutiérrez Gándara Pablo, Lugo Pérez Salvador, Rodríguez Romero Alejandra Berenice, Zárate Salinas Itzel Corina, Colunga Pedraza Iris Jazmín, Galarza Delgado Dionicio Ángel Hospital Universitario «Dr. José Eleuterio González».

Tipo de estudio: Estudio pronóstico

Introducción: Los pacientes con artritis psoriásica (APs) tienen un mayor riesgo cardiovascular (RCV). Se han desarrollado algoritmos de estratificación de RCV para la población general, como las calculadoras de RCV; sin embargo, éstas subestiman el RCV en pacientes con APs. El ultrasonido carotídeo (USc) permite medir el grosor íntima-media carotídeo (GIMc) y la placa carotídea (PC). Objetivo: Reclasificar el riesgo cardiovascular de pacientes con APS mediante el uso del ultrasonido y compararlo con controles. Metodología: Se incluyeron 69 pacientes con APs y 69 controles pareados por edad y comorbilidades. El aumento de GIMc se definió como $\geq 0.9 \mathrm{~mm}$ y la $\mathrm{PC}$ como un estrechamiento $\geq 0.5$ $\mathrm{mm}$ o un $\mathrm{GIMc} \geq 1.2 \mathrm{~mm}$. El ánálisis descriptivo fue realizado con frecuencias (\%) y mediana (q25-q75) y comparaciones con $\chi^{2}$ y U de Mann-Whitney. Se consideró significativa una $p<0.05$. Resultados: Se incluyeron un total de 138 sujetos. Las características clínicas y demográficas se muestran en la Tabla 12.5.1. FRS-lípidos tuvo una mayor prevalencia de reclasificación por aumento del GIMc en los pacientes con APs que en los controles $(p=0.016)$. Se observó que $70.8 \%$ de los pacientes con PC fueron clasificados como de bajo o intermedio RCV y sólo $29.2 \%$ se clasificaron como de alto RCV, según FRS-lípidos ( $p=0.010$ ). Discusión y conclusiones: Ninguna de las calculadoras analizadas estima correctamente el RCV en pacientes con APs. Es aconsejable realizar USc indistintamente de los resultados de éstas para lograr una clasificación y manejo adecuados.

\section{Trabajo ganador del premio Dr. Mariano Ledesma Velasco al Investigador Joven Primer Lugar}

\subsection{La citrulina disminuye los cambios del miocardio debido a enfermedad de Alzheimer en modelo murino}

Pacheco López Alejandro,* Saturno Chiu Guillermo, * Lupercio Mora Karina,* Arellano Escudero Osvaldo,* Palma Guzmán Alam Adrián, ${ }^{\ddagger}$

García De la Torre Paola, ${ }^{\S}$ Almeida Gutiérrez Eduardo*

* Hospital de Cardiología del Centro Médico Nacional Siglo XXI.

‡ Centro de Investigación del Instituto Mexicano del Seguro Social.

$\S$ Hospital de Especialidades del Centro Médico Nacional Siglo XXI. Tipo de estudio: Estudios experimentales (modelo animal, en tejidos o cultivo celular)

Introducción: Los cambios miocárdicos, cerebrales y vasculares están asociados con envejecimiento y demencia. La enfermedad de Alzheimer (EA) es la causa más frecuente de demencia; alteraciones de microvasculatura cerebral conducen a cambios neuroanatómicos, hipoperfusión cerebral y lesión cerebral subclínica; el tejido miocárdico puede verse afectado por EA. La citrulina aumenta el óxido nítrico y mejora la función endotelial. Objetivo: Evaluar el efecto de la suplementación con citrulina en miocardio de modelos murinos con enfermedad de Alzheimer. Metodología: Ensayo ex- 
Figura 12.6.1: Extenso depósito de material eosinófilo amorfo a nivel intersticial probable amiloide $66-100 \%$.
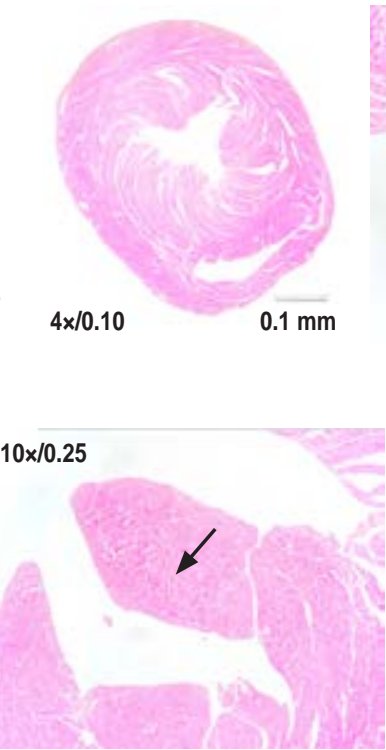
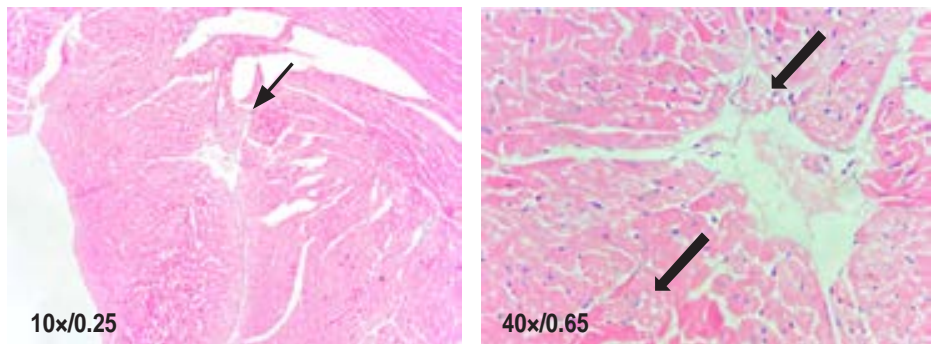

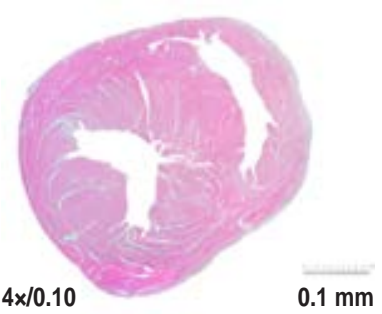

perimental, ratones de 11 meses (tipo salvaje) y 3XTg-AD de ocho meses (transgénicos). Aleatorización: citrulina $1 \mathrm{~g} / \mathrm{kg} /$ día durante tres meses o placebo. Se procesó corazón hematoxilina-eosina. Patólogo en ciego, cambios morfológicos y estructurales. Estadística descriptiva variables absolutas y relativas; Distribución normal: Shapiro-Wilk. Cambios miocárdicos mediante porcentaje cuantitativo en el tejido. Análisis con prueba $U$ de Mann-Whitney y $\chi^{2}$ variables cualitativas. Resultados: En ratones de tipo salvaje, las alteraciones del miocardio

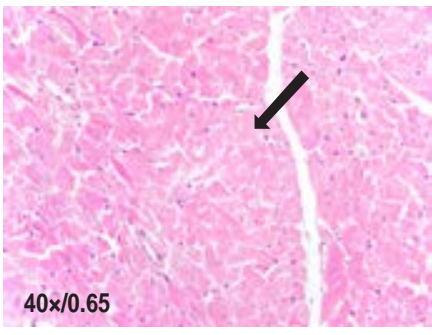

Figura 12.6.2: Acumulación de probable amiloide a nivel perivascular e intersticial $<33 \%$.

fueron significativamente más frecuentes en el grupo placebo $(\mathrm{p}<$ 0.05); mientras tanto, los ratones transgénicos mostraron depósitos de amiloide en 33\% del tejido miocárdico para aquéllos con suplementación con citrulina frente a $66 \%$ en ratones transgénicos con placebo $(p<0.01)$. Discusión y conclusiones: La citrulina mejora los cambios histológicos del tejido miocárdico en modelo murino con enfermedad de Alzheimer y cepas de tipo salvaje. La citrulina podría considerarse tratamiento adyuvante en estos pacientes. 